\title{
MEDIDAS E ESCALAS DE ATITUDE RELATIVA AO PATROCÍNIO
} ESPORTIVO

\author{
Éverton Luís Pellizzaro de Lorenzi Cancellier \\ Doutor em Administração \\ Universidade do Estado de Santa Catarina (UDESC) \\ everton.cancellier@udesc.br
}

Resumo

Objetivo do estudo: o objetivo deste estudo foi investigar medidas e escalas de atitude relativa ao patrocínio esportivo encontradas na literatura internacional.

Metodologia/abordagem: trata-se de uma pesquisa bibliográfica de caráter exploratório e descritivo. A partir de um levantamento bibliográfico realizado na base Ebsco, com a configuração (attitud*) AND (sport*) AND (sponsor*), e após filtragem, foram analisados na integra dezessete estudos sobre o tema em questão.

Originalidade/Relevância: o estudo difere-se de outros principalmente pelo seu foco específico de análise. Destarte, não incide sobre métodos, resultados ou conclusões dos estudos analisado, mas, de outra forma, analisa especificamente as medidas e escalas utilizadas para o alcance dos objetivos das respectivas investigações.

Principais resultados: a partir da análise das medidas e escalas foi possível apontar indicadores comumente utilizados para investigar a atitude relativa ao patrocínio esportivo, destacando-se: as intenções de compra dos produtos do patrocinador; a percepção de sinergia entre a atividade patrocinada e o patrocinador; o grau de envolvimento com a atividade patrocinada; intenção de recomendação (boca-a-boca) do patrocinador; e o reconhecimento da marca patrocinadora.

Contribuições teóricas/metodológicas: o estudo contribui para o campo do conhecimento ao trazer minucias sobre medidas e escalas de atitude relativa ao patrocínio esportivo e identificar os principais indicadores utilizados.

Contribuições sociais / para a gestão: contribui também para a gestão ao possibilitar a visualização de um panorama amplo sobre as diferentes formas de o constructo - atitude em relação ao patrocínio esportivo - ser medido, ampliando assim seu entendimento e, possivelmente, sua utilização.

Palavras-chave: Atitude. Patrocínio Esportivo. Escalas e Medidas.

\section{Cite como \\ American Psychological Association (APA)}

Cárdenas, A. R., Cancellier, E; L. P. de L., \& Tezza, R. (2020). Medidas e escalas de atitude relativa ao patrocínio esportivo. PODIUM Sport, Leisure and Tourism Review, São Paulo, 9(2), 1-14. https://doi.org/10.5585/podium.v9i2.11348. 


\section{MEASURES AND SCALES OF ATTITUDE RELATING TO SPORTS SPONSORSHIP}

\section{Abstract}

Objective of the study: the objective of this study was to investigate measures and scales of attitude related to sports sponsorship found in the international literature.

Methodology /approach: this is an exploratory and descriptive bibliographic search. Based on a bibliographic search carried out on the basis of Ebsco, with the configuration (attitud*) AND (sport*) AND (sponsor*), and after filtering, seventeen studies on the subject in question were analyzed.

Originality / Relevance: the study differs from others mainly because of its specific focus of analysis. Thus, it does not focus on methods, results or conclusions of the studies analyzed, but otherwise, it specifically analyzes the measures and scales used to achieve the objectives of the respective investigations.

Main results: from the analysis of the measures and scales, it was possible to point out indicators commonly used to investigate the attitude regarding sports sponsorship, highlighting: the intentions to purchase the sponsor's products; the perception of synergy between the sponsored activity and the sponsor; the degree of involvement with the sponsored activity; intention of recommendation of the sponsor (mouth to mouth); and the recognition of the sponsoring brand.

Theoretical / methodological contributions: the study contributes to the field of knowledge by bringing details about measures and scales of attitude related to sports sponsorship and identifying the main indicators used.

Social / management contributions: it contributes to management by enabling the visualization of a broad overview of the different forms of the construct - attitude towards sports sponsorship - to be measured, thus expanding its understanding and, possibly, its use.

Keywords: Attitude. Sports Sponsorship. Scales and Measures.

\section{MEDIDAS Y ESCALAS DE ACTITUD RELACIONADAS CON EL PATROCINIO DEPORTIVO}

\section{Resumém}

Objetivo del estudio: el objetivo de este estudio fue investigar medidas y escalas de actitud relacionadas con el patrocinio deportivo que se encuentran en la literatura internacional.

Metodología / enfoque: se trata de una búsqueda bibliográfica exploratoria y descriptiva. Con base en una investigación bibliográfica realizada sobre la base de Ebsco, con la configuración (attitud*) AND (sport*) AND (sponsor*), y después del filtrado, se analizaron diecisiete estudios sobre el tema en cuestión.

Originalidad / Relevancia: el estudio difiere de otros principalmente debido a su enfoque específico de análisis. Por lo tanto, no se centra en los métodos, resultados o conclusiones de los estudios analizados, sino que analiza específicamente las medidas y escalas utilizadas para lograr los objetivos de las investigaciones respectivas.

Resultados principales: a partir del análisis de las medidas y escalas, fue posible señalar los indicadores comúnmente utilizados para investigar la actitud con respecto al patrocinio deportivo, destacando: las intenciones de comprar los productos del patrocinador; la percepción de sinergia entre la actividad 
patrocinada y el patrocinador; el grado de participación en la actividad patrocinada; intención de recomendación (boca a boca) del patrocinador; y el reconocimiento de la marca patrocinadora.

Contribuciones teóricas / metodológicas: el estudio contribuye al campo del conocimiento aportando detalles sobre medidas y escalas de actitud relacionadas con el patrocinio deportivo e identificando los principales indicadores utilizados.

Contribuciones sociales / de gestión: contribuye a la gestión al permitir la visualización de una visión general amplia de las diferentes formas de el constructo - actitud hacia el patrocinio deportivo - medirse, ampliando así su comprensión y posiblemente su uso.

Palabras clave: Actitud. Patrocinio desportivo. Escalas y medidas.

\section{Introdução}

A literatura científica nacional e internacional, a mídia e a própria mobilização envolvida na realização dos megaeventos esportivos sediados no Brasil ao longo da década que se encerra, demonstram o esporte como um fenômeno social consolidado, mundialmente difundido e que movimenta multidões de pessoas - esportistas, praticantes ou espectadores ao redor do globo.

Sabedoras desta realidade, e dos consideráveis montantes de dinheiro envolvidos neste mercado, organizações dos mais variados setores, através da prática do marketing esportivo e especificamente do patrocínio, utilizam o esporte como instrumento/ferramenta/veículo para atingir objetivos organizacionais - criação de posicionamento de marca e ampliação da exposição na mídia, por exemplo (Zardini Filho, 2017; Fernandez, 2011). Sob tal perspectiva, estudos corroboram em relação a influência que o patrocínio esportivo exerce sobre a atitude de consumidores, tornando-se, portanto, um tema relevante aos estudos de marketing e de atitude dos consumidores em geral (Mason, 2005; Zardini Filho, 2017).

Sabe-se, ainda, que a comunidade científica tem investigado a prática do patrocínio sobre diferentes prismas. Neste sentido, investigações já foram conduzidas a partir da perspectiva daquele que patrocina a atividade - o patrocinador. $\mathrm{O}$ foco de tais estudos perpassa os objetivos e/ou motivos do patrocinador em uma ação de patrocínio (Zen \& Machado, 2004; Da Rocha \& Campos, 2010; Pimentel, 2011); o processo de tomada de decisão frente a projetos de patrocínio (Mattar, 2007; da Rocha \& Campos, 2010); motivos da descontinuidade do patrocínio (Almeida, 2009); e estratégias de patrocínio (Ferrari \& Silveira, 2009).

Outro ponto de vista, bastante presente na literatura - e foco deste estudo -, analisa o consumidor da indústria esportiva a partir de sua atitude frente à marca e aos produtos da marca 
patrocinadora. Nesta perspectiva, as investigações costumam explorar principalmente os efeitos que o patrocínio exerce sobre o reconhecimento ou recall da marca patrocinadora, as associações e comportamento potencial perante a marca de patrocinadores, por parte do consumidor (Crescitelli, Mattar \& da Silva, 2005; Zunino, 2006; Grynberg \& da Rocha, 2010; Scharf, 2010).

Frente a este panorama de variedade de metodologias empregadas sobre o tema, o objetivo deste estudo foi investigar as medidas e escalas de atitude em relação ao patrocínio esportivo. Destaca-se não fazer parte do escopo desta pesquisa a análise dos estudos em si, ou dos resultados e conclusões obtidas pelos os mesmos, mas, principalmente, a análise específica das medidas e escalas utilizadas para o alcance dos objetivos das respectivas investigações.

Um estudo nessa dimensão pode contribuir tanto para a comunidade científica, como para os gestores esportivos, na prática da gestão de organizações esportivas, ao possibilitar a visualização de um panorama amplo sobre as diferentes formas de o constructo - atitude relativa ao patrocínio esportivo - ser medido, ampliando assim seu entendimento e, possivelmente, sua utilização. Contribuir para esse entendimento e para a adequada utilização das diferentes escalas e medidas apresentadas pela literatura acadêmica torna-se uma das principais contribuições do presente estudo e justificativa para sua realização.

\section{Referencial Teórico}

\subsection{Patrocínio esportivo e atitude relativa ao patrocínio}

Variados autores conceituam o termo patrocínio - não existindo, assim, um consenso universal a respeito do tema. Não obstante a essa realidade, o patrocínio pode ser entendido, essencialmente, como o fornecimento de recursos (na maioria das vezes financeiros) por uma organização para o suporte de uma determinada atividade (ou evento, atleta, organização esportiva, atividade cultural, social...) com o propósito de associar a imagem da empresa patrocinadora à atividade patrocinada (Contursi, 2003, Keller \& Machado, 2003; Parolini \& Rocco Junior, 2015). O patrocínio esportivo, especificamente, acontece quando os recursos, do ente patrocinador, suportam atletas, atividades, organizações e/ou eventos conexos ao esporte (Pozzi, 1998, Melo Neto, 2003).

O patrocínio de atividades esportivas é um dos principais instrumentos do marketing esportivo e cresce concomitantemente com o desenvolvimento das atividades de marketing que relacionam marcas/organizações/produtos aos esportes. Este crescimento, que permanece 
incrementando os investimentos organizacionais relativos ao esporte, é percebido e relatado por diferentes estudiosos (Azevedo, 2009; Rocha \& Campos, 2010; Parolini \& Rocco, 2015; Moraes, 2017).

Por possibilitar um tipo diferente de interação com os consumidores, o patrocínio esportivo figura como uma interessante ferramenta de comunicação organizacional, construção e posicionamento de marcas na mente dos consumidores (Leocádio et al., 2007; Cárdenas, 2013). Destarte, é comum associar o constructo a diversos outros conceitos relacionados ao marketing - entre eles a atitude. O estudo das atitudes torna-se intrínseco ao processo de marketing esportivo, pois é construto que influência, por exemplo, o comportamento do consumidor em relação à compra de um produto (Mielli \& Mantovani, 2014).

O termo atitude pode ser entendido, essencialmente, como uma predisposição de um sujeito responder de forma consistentemente favorável ou desfavorável em relação um dado objeto, como uma marca, um novo produto, uma propaganda de televisão (Hawkins \& Mothersbaugh, 2018) - e no caso deste estuo, o patrocinador esportivo.

Dentro e fora do contexto esportivo o constructo atitude tem sido analisado sob a luz de diferentes metodologias, escalas e medidas (Porto, 2010; Soares, Deliza \& Gonçalves 2008). No âmbito dos esportes, parte das pesquisas produzidas analisa a atitude concomitantemente a outros constructos relacionados, ou seja, as investigações, de modo geral, costumam observar não somente a atitude relativa ao patrocínio, mas suas relações com o envolvimento ao evento esportivo, com os seus personagens/atores (os esportistas), com a similaridade/sinergia/adequação entre patrocinador e atividade patrocinada (Zardini Filho, 2017).

O presente estudo demonstra em seus resultados esta pluralidade de configurações investigativas.

\section{Metodologia}

Para o alcance do objetivo proposto, foi realizado um levantamento bibliográfico junto à base de dados EBSCOhost Online Research Database (ebscohost.com) com intuito de encontrar um conjunto de trabalhos que versassem sobre o tema atitude relativa ao patrocínio esportivo. A base em questão foi selecionada por contemplar vasto referencial de publicações tanto na área da Administração e Negócios quanto na área dos esportes. 
Desta forma, na plataforma de pesquisa, através da utilização de operadores booleanos, símbolos lógicos e dos termos em inglês relacionados à atitude e ao patrocínio esportivo, foi efetuada a pesquisa - com foco nos resumos/abstract dos trabalhos - com a seguinte formatação/estrutura: (attitud*) AND (sport*) AND (sponsor*).

Destaca-se que nesta base de dados as pesquisas com termos finalizados em asterisco (*) resultam em pesquisa de textos com palavras relacionadas, ampliando assim os resultados da busca. Por exemplo: numa pesquisa cujo termo é "sponsor*" são trazidos aos resultados da pesquisa trabalhos que contenham os termos "sponsor", "sponsorship", "sponsoring”. Não foi usado filtro em relação ao período temporal.

A pesquisa inicial - (attitud*) AND (sport*) AND (sponsor*) -, realizada em 2018, trouxe à tona 201 textos que após filtragem com a opção "texto completo" foram reduzidos a 84 trabalhos. Através da leitura do título e do resumo de todos os 84 textos, foram excluídos os trabalhos que não se alinhavam ao escopo da presente pesquisa (embora possuíssem as palavras-chave em seus resumos, não abordavam o tema objetivado por este estudo), os textos duplicados, e, ainda, aqueles que não se tratavam de pesquisas científicas (por exemplo: reportagens jornalísticas, informativos, editoriais, etc.). Por fim, ainda que tangenciassem o tema em questão, foram excluídos os estudos que não utilizavam ou não apresentavam escalas e medidas para a análise do constructo (como investigações com abordagem qualitativa).

Ao final, restaram 17 estudos pertinentes ao tema e ao objetivo proposto, que foram, então, analisados integralmente para entendimento das medidas e escalas utilizadas.

\section{Resultados e discussão}

O Quadro 1 apresenta os estudos identificados na busca da base de dados, demonstrando os autores das pesquisas e o ano de publicação de cada trabalho, em ordem cronológica. 
Quadro 1 - Autores e títulos dos estudos encontrados na base de dados

\begin{tabular}{|c|c|}
\hline Autor(es) & Título do trabalho \\
\hline Mcdaniel \& Heald (2000) & $\begin{array}{l}\text { Young consumers' responses to event sponsorship advertisements of } \\
\text { unhealthy products: Implications of schema-triggered affect theory. }\end{array}$ \\
\hline Kuzma et al. (2003) & $\begin{array}{l}\text { Negative corporate sponsor information: The impact on consumer } \\
\text { attitudes and purchase intentions. }\end{array}$ \\
\hline Amato et al. (2005) & An exploratory investigation into NASCAR fan culture. \\
\hline $\begin{array}{l}\text { Koo, Quarterman \& } \\
\text { Flynn (2006) }\end{array}$ & $\begin{array}{l}\text { Effect of perceived sport event and sponsor image fit on consumers' } \\
\text { cognition, affect, and behavioral intentions. }\end{array}$ \\
\hline $\begin{array}{l}\text { Alexandris, Tsaousi \& } \\
\text { James (2007) }\end{array}$ & $\begin{array}{l}\text { Predicting sponsorship outcomes from attitudinal constructs: The case } \\
\text { of a professional basketball event. }\end{array}$ \\
\hline Alay (2008) & $\begin{array}{l}\text { Female consumers'evaluations of sponsorship and their response to } \\
\text { sponsorship. }\end{array}$ \\
\hline $\begin{array}{l}\text { Dees, Bennett \& Villegas } \\
(\text { (2008) }\end{array}$ & $\begin{array}{l}\text { Measuring the effectiveness of sponsorship of an elite intercollegiate } \\
\text { football program. } 2008 \text {. }\end{array}$ \\
\hline $\begin{array}{l}\text { Danylchuk \& Macintosh } \\
\text { (2009) }\end{array}$ & $\begin{array}{l}\text { Food and non-alcoholic beverage sponsorship of sporting events: the } \\
\text { link to the obesity issue. }\end{array}$ \\
\hline Park et al. (2011) & $\begin{array}{l}\text { Consumer attitudes toward the Paralympic Games and purchase } \\
\text { intentions toward corporate sponsors of the Paralympic Games: market } \\
\text { segmentation strategy. }\end{array}$ \\
\hline Tobin et al. (2012) & $\begin{array}{l}\text { Support for breaking the nexus between alcohol and community sports } \\
\text { settings: Findings from the VicHealth Community Attitudes Survey in } \\
\text { Australia. }\end{array}$ \\
\hline $\begin{array}{c}\text { Eagleman \& Krohn, } \\
(\mathbf{2 0 1 2})\end{array}$ & $\begin{array}{l}\text { Sponsorship awareness, attitudes, and purchase intentions of Road } \\
\text { Race Series participants. }\end{array}$ \\
\hline $\begin{array}{l}\text { Mckelvey, Sandler \& } \\
\text { Snyder (2012) }\end{array}$ & $\begin{array}{l}\text { Sport participant attitudes toward ambush marketing: An exploratory } \\
\text { study of ING New York City Marathon runners. }\end{array}$ \\
\hline Macintosh et al. (2012) & $\begin{array}{l}\text { The role of mega-sports event interest in sponsorship and ambush } \\
\text { marketing attitudes. }\end{array}$ \\
\hline Dhurup \& Rabale (2012) & $\begin{array}{l}\text { Spectators' perceptions of official sponsors in the FIFA } 2010 \text { World } \\
\text { Cup and purchase intentions of sponsors products or brands. }\end{array}$ \\
\hline Close \& Lacey (2013) & $\begin{array}{l}\text { Fit Matters? Asymmetrical Impact of Effectiveness for Sponsors and } \\
\text { Event Marketers. }\end{array}$ \\
\hline $\begin{array}{l}\text { Reams, Eddy \& Cork } \\
\text { (2015) }\end{array}$ & Points of attachment and sponsorship outcomes in an individual sport. \\
\hline Zaharia et al. (2016) & No More'Good'Intentions: Purchase Behaviors in Sponsorship \\
\hline
\end{tabular}

Fonte: Elaborado pelos autores.

Tendo em vista o panorama geral de trabalhos envolvidos nesta pesquisa, apresenta-se a seguir uma breve contextualização dos estudos e síntese dos instrumentos de medidas e escalas utilizadas em cada investigação. 


\subsection{Estudos, escalas e medidas sobre atitude relativa ao patrocínio esportivo}

\section{O estudo de McDaniel \& Heald (2000)}

O estudo destes autores teve como pano de fundo a discussão relativa ao patrocínio de eventos esportivos por produtos considerados prejudiciais à saúde, insalubres ou inseguros (como fast-food, cigarros, bebidas alcoólicas, etc.).

A metodologia empregada envolveu uma pesquisa experimental baseada na teoria de efeitos desencadeados, onde foram usadas variações em peças de publicidade impressas de produtos patrocinadores de eventos esportivos, para verificar a atitude dos sujeitos pesquisados. A parte principal do estudo tinha três etapas e a amostra contou com 215 estudantes de graduação de uma universidade dos Estados Unidos.

Inicialmente os estudantes eram convidados a responder a primeira parte do questionário que buscava identificar o envolvimento e atitude que os participantes possuíam em relação a três eventos esportivos (PBA Bowler's Tour, NHL Hockey sobre gelo e Jogos Olímpicos); e a duas categorias de produtos (cigarros e fast-food). Posteriormente os pesquisados eram convidados a avaliar a adequação/inadequação de protótipos de publicidade de produtos patrocinadores de eventos esportivos, para futuras campanhas promocionais (estas peças haviam sido criadas/adaptadas apenas para a pesquisa, mas utilizavam marcas de empresas reais e conhecidas). Por fim, os pesquisados respondiam à itens que buscavam identificar possíveis alterações da atitude (efeitos desencadeados) da exposição à publicidade.

O instrumento de medida da pesquisa utilizava-se de escala semânticas de 7 pontos para as diferentes dimensões analisadas. Na primeira parte do questionário dez itens buscavam identificar o envolvimento e três itens identificavam a atitude dos pesquisados. A avaliação das peças publicitárias, por parte dos pesquisados continha oito itens (entre eles: desfavorável/favorável, ruim/bom, irritante/não irritante, bem produzido/mal produzido, etc.). A última parte do questionário apresentava ainda três itens sobre intenção de compra (atitude) e seis itens de avaliação geral dos anúncios observados (ancoradas por mal combinado/bem combinado, apropriado/inapropriado, etc.) além de itens de identificação do gênero, idade e raça dos participantes da investigação.

\section{O estudo de Kuzma et al. (2003)}


De maneira semelhante à pesquisa de McDaniel \& Heald (2000), o estudo experimental desenvolvido por Kuzma et al. (2003) teve o objetivo de examinar o impacto de informações negativas sobre um patrocinador esportivo, na atitude e intenção de compra de potenciais consumidores.

A investigação foi desenvolvida no contexto de patrocínio do departamento de esportes de uma universidade dos Estados Unidos, em três partes sequenciais. Inicialmente os sujeitos preenchiam um questionário que buscava medir as atitudes em relação ao patrocinador e intenções de compra de produtos do patrocinador (no caso a empresa Nike).

$\mathrm{Na}$ segunda parte do estudo os sujeitos assistiam a um filme com cenas do time de futebol americano da universidade, com o logotipo do patrocinador (Nike) disposto nas cenas, uma breve descrição dos recursos financeiros contratados entre a universidade/patrocinador; e, também, a cenas de um protesto estudantil contra condições de trabalho questionáveis em fábricas do patrocinador fora do país.

$\mathrm{Na}$ terceira parte do estudo os sujeitos completavam o questionário que buscava medir novamente as atitudes em relação ao patrocinador e intenção de compra. Embora o instrumento não seja descrito detalhadamente no trabalho, os resultados do experimento demonstraram influência negativa nas atitudes e intenções de compra do patrocinador.

\section{Estudo de Amato (2005)}

O trabalho desenvolvido por este autor examinou os fãs da NASCAR (National Association for Stock Car Auto Racing/EUA). O estudo teve foco sobre as atitudes e comportamentos dos fãs/espectadores sobre o esporte - Stock Car -, não tendo objetivos específicos de análise sobre a atitude em relação aos patrocinadores do esporte.

Não obstante, pequena parte do instrumento utilizado (3 itens) buscava medir a atitude relativa ao patrocínio do esporte, medindo suas respostas por meio de escala likert de cinco pontos. Os itens da escala eram relacionados ao uso de produtos de patrocinadores dos pilotos favoritos; atitude perante produtos dos patrocinadores de pilotos que não gostava/não tinha afinidade; bem como reconhecimento do patrocinador do piloto favorito.

\section{O Estudo de Koo, Quarterman \& Flynn (2006)}

A escala desenvolvida neste estudo teve o objetivo de entender o efeito da percepção do evento esportivo e a sinergia/adequação de seu patrocinador na atitude de espectadores do 
evento. A pesquisa foi desenvolvida em dois estágios. Um pré-teste com 162 participantes foi realizado anteriormente ao teste principal que incluiu 452 espectadores do College Bowl Championship Series de 2003, nos Estados Unidos.

Segundo os autores, o pré-teste facilitou a identificação de um conjunto de "adjetivos de personalidade" que descrevessem o evento esportivo (College Bowl Championship Series) e um conjunto de marcas patrocinadoras consideradas adequadas ao evento, pelos respondentes. A principal fase do teste foi usada para examinar os efeitos da percepção de imagem do evento no reconhecimento de marcas patrocinadoras e atitude em relação à marca patrocinadora.

No instrumento, para identificar o grau de sinergia/adequação do patrocinador ao evento foi utilizado uma escala de 6 itens, sendo que os itens da escala eram de resposta do tipo escala Likert de sete pontos. Atitude dos espectadores foi medida usando 3 itens (com respostas em escala semântica de sete pontos). Para o reconhecimento da marca patrocinadora foi utilizado apenas um item; e as intenções de compra foram medidas por 2 itens (escala semântica de sete pontos).

\section{O estudo de Alexandris, Tsaousi \& James (2007)}

A escala desenvolvida neste estudo foi baseada em estudos anteriores (não especificados pelos autores) e objetivou testar o grau em que três efeitos comumente associados ao patrocínio - quais sejam: imagem do patrocinador, intenção de fazer recomendações boca-a-boca sobre o patrocinador e as intenções de compra - podem ser estimados por três constructos de atitude: atitude em relação ao evento, envolvimento com a atividade esportiva e crenças sobre patrocínio.

A pesquisa foi desenvolvida durante um evento de basquete profissional realizado na Grécia (All-star Basketball Game), tendo sido aplicado um questionário aos espectadores do evento e obtendo-se 257 respostas válidas.

O instrumento do estudo coletava informações sobre (1) o perfil dos respondentes sexo, idade, estado civil, e educação; (2) Reconhecimento do patrocinador oficial do evento (um item questionando qual era o patrocinador, que no caso da pesquisa era apenas um); (3) Atitude em relação ao evento (três itens); (4) envolvimento com atividade esportiva (seis itens); (5) Crenças sobre patrocínio (quatro itens); (6) imagem do patrocinador (quatro itens); e (7) Intenções de compra do patrocinador (3 itens) e intenção de recomendação boca-a-boca do patrocinador (1 item). 


\section{O estudo de Alay (2008)}

Alay (2008) pesquisou especificamente a atitude de mulheres (413 estudantes universitárias) em relação ao patrocínio de um evento esportivo. O instrumento de pesquisa foi um questionário de avaliação de patrocínio desenvolvido anteriormente pelo próprio autor, com 55 itens (respostas em escala Likert de sete pontos).

A parte introdutória do instrumento é composta por questões sobre perfil dos sujeitos e sobre seu nível de participação no evento esportivo. Este nível de participação dos respondentes foi medido por um único item de autoavaliação - que melhor descrevesse a participação do respondente no evento.

A principal parte do questionário tem duas seções e busca medir (1) a percepção do participante do evento sobre o patrocínio e (2) resposta/atitude do participante ao patrocinador.

\section{O estudo de Dees, Bennett \& Villegas (2008)}

A escala desenvolvida no estudo buscou avaliar o patrocínio de um programa universitário de futebol americano profissional. Durante a construção do instrumento de pesquisa os itens do questionário foram avaliados por especialistas (professores universitários e, também, por estudantes de uma disciplina de pesquisa) que foram convidados a julgar a validade de conteúdo, comentar a relevância, representatividade e clareza de cada um dos itens, fornecendo sugestões para melhoria do instrumento. Foi, também, realizado teste piloto ( $\mathrm{N}=49$ ) em um evento de mesmo porte do qual a pesquisa iria ser realizada.

O instrumento final da pesquisa - questionário - foi aplicado de forma online a 394 sujeitos e era composto por 16 itens divididos igualmente em quatro dimensões - (1) atitudes em relação ao patrocinador, (2) boa intenção do patrocinador, (3) intenção de compra de produtos do patrocinador e (4) envolvimento com o esporte - além de uma seção incluindo cinco itens relacionados ao perfil dos sujeitos pesquisados.

\section{O estudo de Danylchuk \& Macintosh (2009)}

No estudo realizado por estes autores, cujo objetivo era examinar a opinião de consumidores esportivos em relação à adequação de diferentes tipologias de patrocinadores esportivos (tabaco, bebidas alcoólicas, bebidas não alcoólicas, alimentos fast-foods, não-fastfood), foi desenvolvida uma pesquisa de caráter misto - quantitativo e qualitativo, 
respectivamente sequenciais. As metodologias empregadas no estudo foram a aplicação de um survey $(\mathrm{N}=253)$ e, posteriormente o desenvolvido um trabalho com 12 grupos focais.

A fase quantitativa da pesquisa fora conduzida com base em um instrumento com duas partes principais. Uma delas com questões relativas ao perfil do respondente (gênero, idade, formação educacional, etc.); e outra com três questões principais, voltadas à questão do patrocínio. A primeira questão buscava identificar o grau de concordância dos participantes perante a adequação de variadas categorias de patrocinadores a um evento esportivo: empresas de tabaco, bebidas alcoólicas, bebidas não alcoólicas, alimentos fast-foods, não-fast-food, etc. Para esta parte da pesquisa as respostas eram dadas em escala Likert de cinco pontos. Uma segunda pergunta aberta solicitou aos participantes que fornecessem razões para as suas diferenças de opinião entre as diferentes categorias de patrocinadores. Por fim, a terceira questão continha uma lista variada de vinte possíveis tipos de patrocinadores de eventos esportivos, sendo solicitado aos participantes que avaliassem os três tipos mais apropriados e os três menos apropriados (a lista continha além das tipologias de patrocinadores já mencionadas anteriormente, instituições financeiras, empresas de telefonia, entre outras).

\section{O estudo de Park et al. (2011)}

O objetivo principal do estudo era analisar as atitudes dos consumidores em relação aos Jogos Paraolímpicos e suas intenções de compra relativas às marcas e produtos de patrocinadores dos Jogos. Além deste, o estudo igualmente objetivou investigar o efeito das variáveis demográficas e psicográficas sobre as atitudes dos consumidores. Os dados foram coletados a partir de das respostas de 209 estudantes universitários de duas grandes universidades do Centro-Oeste americano.

O instrumento do estudo foi um questionário (impresso) contendo em três partes principais: (1) informação demográficas/de perfil do respondente, (2) a percepção dos consumidores em relação à responsabilidade social das organizações patrocinadoras e (3) as atitudes e intenções de compra dos consumidores em relação aos patrocinadores dos Jogos Paraolímpicos.

Cinco itens foram usados para medir as atitudes em relação aos Jogos Paraolímpicos por exemplo: "os Jogos Paraolímpicos serão um evento esportivo emocionante de assistir", ou "os Jogos Paraolímpicos promoverão uma imagem mais positiva das pessoas com deficiência física". As intenções de compra foram avaliadas por um item único ("Quando o preço e 
qualidade são iguais, eu vou mudar para marcas ou comprar produtos de as empresas que patrocinam os Jogos Paraolímpicos"). Para medir a percepção dos consumidores em relação à responsabilidade social das organizações patrocinadoras dos jogos foi utilizado um item único. Todos os itens do instrumento eram respondidos com escala Likert de cinco pontos.

\section{O estudo de Tobin et al. (2012)}

O objetivo da pesquisa de Tobin et al. (2012) fora descrever a atitude em relação ao álcool em comunidades de prática esportivas. Mas além deste, outros objetivos fizeram parte da pesquisa realizada, como: a concordância/apoio para a alteração das configurações da relação álcool e comunidade de prática esportiva, a intenção de aumentar a participação em clubes esportivos comunitários de eventuais alterações e, por fim, a percepção da adequação do patrocínio esportivo por parte de empresas de bebidas alcoólicas.

A pesquisa foi conduzida tendo como sujeitos investigados adultos residentes no estado de Victória/Austrália. Os dados foram coletados por telefone, onde 3677 ligações foram realizadas para serem obtidas 1500 respostas válida para o survey.

O instrumento continha partes relacionadas à identificação do perfil sócio demográfico dos participantes; dos hábitos de consumo de álcool; do envolvimento e intenção de participação em comunidades esportivas locais; porém apenas um item relacionado à concordância em relação à adequação do patrocínio de comunidade esportivas por parte da indústria de bebidas alcoólicas.

\section{O estudo de Eagleman \& Krohn (2012)}

Esta pesquisa apresentou uma escala com finalidade de relacionar o reconhecimento de patrocinadores, as atitudes em relação aos mesmos, e intenção de compra dos produtos dos patrocinadores, de participantes de um evento esportivo - O Road Race Series 2011, realizado em diferentes cidades nos Estados Unidos. Na pesquisa, buscou-se identificar relações entre as variáveis mencionadas com base em dados demográficos, nível de identificação com o evento e uso do website/ou página do Facebook do evento.

$\mathrm{O}$ instrumento foi elaborado com base em pesquisas anteriores sobre patrocínio, sendo que além de questões pertinentes ao perfil do respondente, a pesquisa também perguntou aos sujeitos sobre o seu envolvimento com a série de corridas e uso do site o/ou página do Facebook. 
Com o intuito de determinar reconhecimento dos respondentes quanto aos patrocinadores do evento um item do questionário foi construído considerando estudos anteriormente realizados por outros autores, em que "patrocinadores oficiais do evento" e empresas "não patrocinadoras do evento" eram apresentados em ordem aleatória, tendo os respondentes que identificar patrocinadores oficiais do evento.

Para medir as atitudes dos respondentes em relação aos patrocinadores e suas intenções de compra dos produtos dos mesmos, três itens do instrumento foram medidos usando uma escala Likert de sete pontos. Os itens eram: (1) Eu acredito que é bom para as empresas estarem patrocinando o evento. (2) Minhas atitudes em relação a uma empresa são influenciadas por elas patrocinarem o evento. (3) Eu sou mais propenso a comprar produtos ou serviços de uma empresa se ela é uma patrocinadora do evento.

Por fim, o instrumento apresentava a questão sobre quão frequente o sujeito pesquisado havia comprado produtos de patrocinadores do evento no ano anterior (escala de sete pontos, com 1 indicando "nunca" a 7 indicando "muitas vezes". O instrumento continha, ainda, três itens que buscavam medir o nível de identificação com o evento e dois itens que identificavam a frequência de utilização do website/facebook do evento esportivo.

\section{O estudo de Mckelvey, Sandler \& Snyder (2012)}

Sob uma perspectiva peculiar sobre a atitude em relação a patrocinadores esportivos, a escala desenvolvida neste estudo analisou a atitude em relação a empresas que realizam a prática de marketing de emboscada. Em outras palavras, empresas que, por exemplo, buscam ter suas marcas associadas a eventos esportivos sem, no entanto, estarem patrocinando efetivamente/oficialmente os eventos em questão.

No instrumento de medida havia sete itens específicos que buscavam medir a atitude do participante do evento esportivo - no caso participantes das edições de 2005 e 2008 da "ING New York City Marathons" - perante empresas que realizassem o marketing de emboscada. Como exemplos de itens utilizados no instrumento, podem ser citados: "é justo empresas associarem suas marcas sem serem patrocinadores oficiais do evento"; e "se uma empresa tenta se associar com o evento eu serei menos propenso a quere comprar seu produto ou serviço no futuro". As respostas aos itens eram dadas em escala do tipo Likert de sete pontos.

\section{O estudo de MacIntosh et al. (2012)}


A pesquisa coletou, em quatro diferentes cidades do Canadá, dados de 619 sujeitos sobre o interesse em um megaevento esportivo (no caso da pesquisa, os Jogos Olímpicos de Inverno de 2010/Vancouver) e como esse interesse seria determinante da atitude em relação ao patrocinador/patrocínio do evento, intenção de compra de produtos do patrocinador e em relação ao marketing de emboscada.

O instrumento utilizado apresenta inicialmente questionamentos sobre o interesse que os respondentes possuem no evento esportivo em questão ou em outros megaeventos de igual magnitude (como Copa do Mundo de Futebol, Superbowl, etc.). A parte principal do instrumento apresenta 26 itens - divididos em seis dimensões - que buscam identificar (através de escala Likert de cinco pontos) atitudes em relação ao patrocinador do evento e ao marketing de emboscada. São exemplos de questionamentos existentes no instrumento - por exemplo se os respondentes são capazes de distinguir entre patrocinadores oficiais e "não-oficiais"; além de pedir aos respondentes que listem alguns dos patrocinadores oficiais do evento esportivo. Questões relacionadas ao perfil do pesquisado são questionados ao final do instrumento - sexo, idade, nível de escolaridade, renda familiar, etc.

\section{O estudo de Dhurup \& Rabale (2012)}

O objetivo do estudo foi determinar a percepção dos espectadores da Copa do Mundo de Futebol de 2010 em relação aos patrocinadores oficiais do evento, bem como as intenções de compra de produtos dos patrocinadores.

A pesquisa foi realizada na cidade de South Gauteng/África do Sul, com 462 respondentes (espectadores, fãs e seguidores do evento). A escala utilizada na pesquisa consistia em um questionário autoadministrado dividido em seções que mediam - por meio de escala Likert de cinco pontos - as percepções dos espectadores sobre o patrocínio do megaevento e as intenções de compra dos produtos de seus patrocinadores. Existia ainda uma seção que coletava informações sobre o perfil dos entrevistados, incluindo sexo, idade, etnia, comportamentos gerais relativos à audiência de jogos e eventos esportivos, frequência de jogos nos estádios, etc.

O questionário foi pré-testado com um amostra $(\mathrm{N}=10)$ utilizando da metodologia "bola de neve". Com a realização de alterações (de redação, sequência e layout) advindas do préteste, um estudo piloto do questionário foi realizado junto de uma amostra por conveniência $(\mathrm{N}=40)$. $\mathrm{O}$ instrumento final contou com 25 itens divididos em 5 dimensões. 


\section{O estudo de Close \& Lacey (2013)}

O estudo destes autores investigou com profundidade a sinergia/adequação entre patrocinadores e eventos esportivos, apresentando um instrumento que envolvia diferentes aspectos em relação à atitude relativa ao patrocínio de um evento esportivo.

Baseado em 1.615 respostas de campo, o trabalho fundamentalmente buscou evidenciar o impacto da não sinergia, ou seja, a não congruência/adequação entre evento e patrocinador em um evento de grande porte de ciclismo profissional.

O questionário foi aplicado durante sete dias de evento em diferentes cidades onde o tour de ciclismo aconteceu, tendo participado 1739 sujeitos (1615 repostas válidas).

O instrumento construído para a pesquisa foi baseado em instrumentos de outros autores, possuindo 29 itens divididos equilibradamente em 8 dimensões. Os cinco itens para medir a sinergia entre evento e patrocinador do evento foram adaptados de Speed e Thompson (2000) e alteradas para adequarem-se o contexto para este estudo.

\section{O estudo de Reams, Eddy \& Cork (2015)}

O estudo destes autores objetivou analisar quais questões relacionadas a um esporte individual seriam mais eficazes para determinar a eficácia do patrocínio. A pesquisa foi desenvolvida no contexto do UFC - Ultimate Fighting Championship. As questões relacionadas ao esporte medidas foram o apego/afeto/ligação ao campeonato/evento; ao lutador; ao esporte propriamente dito e ao nível de competição; e a eficácia do patrocínio foi medida pela atitude em relação aos patrocinadores do UFC; patrocinadores pessoais dos lutadores; intenções de compra do patrocinador do UFC; e intenções de compra dos patrocinadores pessoais dos lutadores.

A pesquisa foi realizada de forma online durante duas semanas, em setembro de 2014, e recolheu 177 respostas válidas. O instrumento inicialmente solicitava que os respondentes identificassem o seu lutador favorito no UFC. Seguidamente, por se tratar de um questionário online, o sujeito era direcionado a questões relacionadas especificamente ao lutador selecionado como preferido. Com utilização de escala Likert de sete pontos, o instrumento contou com 36 itens que buscavam mediar o apego/ligação que o respondente tinha com o esporte (MMA); com o esporte profissional; com o UFC (que é a mais famosa liga mundial deste esporte); com um lutador favorito; bem como medir as atitudes (intenção de compra e de recomendação de produtos) em relação aos patrocinadores do UFC e patrocinadores pessoais dos lutadores. 


\section{O estudo de Zaharia et al. (2016)}

O estudo levanta um questionamento sobre pesquisas realizadas no âmbito da intenção de compra. Acreditam, os autores, que uma avaliação mais precisa da eficácia do patrocínio esportivo adviria através de medição dos comportamentos reais de compra e não somente de intenções.

Neste sentido, o estudo desenvolvido teve como objetivo examinar o patrocínio esportivo incluindo conhecimento do patrocinador, sinergia/adequação com a atividade patrocinada, atitude em relação ao patrocinador, compras anteriores, intenção de compra e especificamente sobre as compras reais - ou seja, compras efetivamente realizadas durante um período de tempo anterior.

Para testar o modelo proposto, foi realizada uma pesquisa através de um questionário online longitudinal conduzido com fãs americanos de futebol, sobre a eficácia do patrocinador do Chelsea Futebol Clube (Inglaterra) - cujo patrocinador era a SANSUNG (patrocinador da camiseta).

A pesquisa foi desenvolvida em duas partes principais. Na primeira fase do estudo, com duração de 22 semanas, foi realizada uma pesquisa (questionários online) para examinar (1) consciência sobre patrocínio (um item), (2) a sinergia/adequação do patrocínio (quatro itens), (3) atitude em relação ao patrocinador (três itens), (4) intenção de compra (três itens) e (5) compras anteriores de produtos do patrocinador (um item). 219 questionários válidos foram devolvidos. $\mathrm{O}$ instrumento on-line fora composto por itens adaptados de estudos anteriores realizados no contexto de patrocínio esportivo para medir as variáveis/dimensões já mencionadas (reconhecimento, adequação...).

Cabe mencionar que no instrumento, especificamente no item relativo ao reconhecimento da marca patrocinadora, por exemplo, os respondentes tinham apenas 20 segundos para introduzir o nome correto do patrocinador antes que fossem direcionados para a próxima pergunta. Tal procedimento, segundo os autores, buscava evitar que o respondente buscasse auxílio para responder ao item. Além disso, um item do instrumento testava a atenção do respondente durante a aplicação do questionário, ao solicitar que o respondente clicasse obrigatoriamente em "concordo fortemente" em determinada afirmativa - apenas para testar a atenção do respondente. Os itens do questionário eram compostos por itens de resposta tipo Likert de dez pontos. 
A segunda fase do estudo envolveu uma pesquisa "follow-up" - ou seja, realizada com sujeitos participantes da primeira parte da pesquisa (que informaram seu e-mail na fase anterior) - em uma data posterior. Nesta segunda etapa 120 questionários válidos foram devolvidos, que buscaram, fundamentalmente, identificar as compras reais, realizadas no intervalo entre a primeira e segunda fases do estudo.

Tendo em vista o panorama geral de medidas e escalas apresentadas até aqui, é possível perceber que os estudos desenvolvidos têm explorado de maneira bastante heterogênea o tema atitude relativa ao patrocínio esportivo, ao longo dos anos. Neste sentido, distintos contextos esportivos têm sido foco das pesquisas realizadas sobre atitude, partindo de esportes individuais - como o ciclismo (Close \& Lacey, 2013), o UFC (Reams, Eddy \& Cork, 2015) e o automobilismo (Amato, 2005) -, até os esportes coletivos - como basquete (Alexandris, Tsaousi \& James, 2007) e futebol americano (Dees, Bennett \& Villegas (2008).

Se por um lado, a atitude relativa ao patrocínio esportivo é investigada em contextos mais regionais ou locais como no caso de atividades esportivas/eventos universitários (Kuzma et al., 2003; Dees, Bennett \& Villegas, 2008); por outro lado, também é possível encontrar estudos que analisam-na sob a perspectiva de expectadores de megaeventos de abrangência mundial, como a Copa do mundo de Futebol (Dhurup \& Rabale, 2012) e as Paraolimpíadas (Park et al., 2011).

O próprio foco de análise apresenta-se plural entre as investigações realizadas, partindo da análise de espectadores de um evento específico (Koo, Quarterman \& Flynn, 2006; Alexandris, Tsaousi \& James, 2007); passando pelo fã de determinado esporte ou clube esportivo (Amato, 2005; Zaharia et al., 2016), e até mesmo o próprio participante/atleta que vivencia uma corrida/maratona (Mckelvey, Sandler \& Snyder, 2012). Tais análises peculiares demonstram a diversidade de perspectivas empregadas nas investigações sobre o tema.

Portanto, como pode ser notado, não há um único modelo de pesquisa ou mesmo instrumento de medida/escala que contemple as diferentes possibilidades de investigar o constructo atitude relativa ao patrocínio esportivo. Isso porque a própria atitude relativa às marcas e produtos pode estar associada a diferentes outros conceitos afetos ao tema, como demonstraram os trabalhos analisados. Em outras palavras, a predisposição de um sujeito responder de forma favorável ou desfavorável em relação ao patrocinador ou seus produtos é influenciado por múltiplas questões, o que possibilita ampla diversidade de configurações investigativas. 
Enfim, é adequado ser frisado que de acordo com a conveniência e as necessidades dos estudos que são e serão desenvolvidos, novas medidas, escalas e métodos podem ser utilizados, na intenção de aferir indicadores, resultados e conclusões ainda mais condizentes com as realidades investigadas. Destarte, é pertinente considerar a diversidade de medidas e escalas detalhadas no presente estudo e alicerçar-se, obviamente, nos princípios metodológicos de pesquisas científicas. Nesta direção é possível considerar, por exemplo, o trabalho de Hair, Silva Gabriel, Silva \& Braga Junior (2019), que explora, de modo detalhado, elementos fundamentais de desenvolvimento e validação de escalas de mensuração de atitudes.

\section{Considerações finais}

O entendimento das diversas possibilidades de medidas e escalas de um constructo - no caso desta pesquisa, a atitude em relação ao patrocínio esportivo - permite ampliar não somente a compreensão sobre o tema em foco, mas possibilita adequar o instrumento utilizado aos propósitos pretendidos às pesquisas científicas que são conduzidas e a própria prática da gestão em organizações envolvidas com patrocínio esportivo.

Reportando-se ao objetivo da pesquisa, o qual pretendeu investigar medidas e escalas de atitude relativa ao patrocínio esportivo, pode-se chegar as seguintes considerações:

A atitude em relação ao patrocínio esportivo é um constructo que pode, e tem sido investigado com diferentes propósitos e especificidades pela comunidade científica internacional. Em consequência dos diferentes contextos que são explorados e da variedade de objetivos almejados concomitantemente nas pesquisas, resulta-se em uma gama variada de escalas e medidas que são encontradas nos estudos produzidos.

A atitude do consumidor esportivo (seja ele o praticante de atividades esportivas, espectadores de eventos esportivos, torcedores/fãs de um clube esportivo, ou outras variadas formas de consumir o esporte) em relação ao patrocínio deve ser entendida, basicamente, como a predisposição de um sujeito responder de forma favorável ou desfavorável em relação ao patrocinador e seus produtos. Neste sentido, a partir da análise dos estudos pode-se constatar que: as intenções de compra dos produtos do patrocinador; a percepção de sinergia entre a atividade patrocinada e o patrocinador; o grau de envolvimento com a atividade patrocinada; intenção de recomendação (boca-a-boca) do patrocinador; o reconhecimento da marca patrocinadora, a percepção de imagem, de boa intenção, de responsabilidade social e comprometimento com o esporte do patrocinador, são indicadores comumente utilizados para 
investigar o constructo em questão e, portanto, uma das principais contribuições do presente estudo.

De forma dependente aos objetivos almejados por cada investigação (seja ela científica ou de suporte para tomada de decisão na gestão no contexto organizações vinculadas ao esporte), as distintas escalas e medidas apresentadas neste estudo possibilitam um amplo panorama de possibilidades de configurações para entender o constructo atitude relativa ao patrocínio esportivo.

A partir deste panorama geral, é imprescindível que para cada caso e objetivo almejado seja realizado um mergulho mais profundo sobre metodologias, instrumentos, escalas e medidas utilizadas, bem como adaptação destes aos contextos particulares dos pesquisadores/gestores a fim de construir instrumentos confiáveis que efetivamente encontrem aquilo que o pesquisador procura.

Como sugestão para futuras pesquisas sugere-se a identificação das relações estabelecidas entre os estudos e instrumentos utilizados, ou seja, quais são os autores e instrumentos que baseiam a construção dos instrumentos mais recentemente usados, ou quais são as escalas mais replicadas nas pesquisas e na tomada de decisão empresarial. É possível também ampliar ou restringir a busca de pesquisas, dando um foco mais regional - quais escalas e medidas são mais replicadas em determinado país ou continente? Sugere-se igualmente revisões sistemáticas que abarquem os métodos adotados e os resultados obtidos em cada trabalho. Estudos nacionais/brasileiros podem ser foco de futuras investigações. Tais respostas, aliadas aos achados pelo presente estudo, contribuirão ainda mais para o entendimento da temática aqui levantada.

\section{Referências}

Alay, S. (2008). Female consumers' evaluations of sponsorship and their response to sponsorship. South African Journal for Research in Sport, Physical Education and Recreation, 30(2), 15-29.

Alexandris, K., Tsaousi, E., \& James, J. (2007). Predicting sponsorship outcomes from attitudinal constructs: The case of a professional basketball event. Sport Marketing Quarterly, 16(3), 130.

Almeida, R. G. M. D. (2009). Analise da descontinuidade do patrocinio esportivo em clubes de futebol no Brasil. 
Amato, C. H., Peters, C. L. O., \& Shao, A. T. (2005). An exploratory investigation into NASCAR fan culture. Sport Marketing Quarterly, 14(2).

Azevedo, P. H. ( 2009). O esporte como negócio: Uma visão sobre a gestão do esporte nos dias atuais. Revista Estudos, Goiânia, 36 (9/10), 929-939.

Cárdenas, A. R., Costa, J. I. P., \& Mondo, T.S. (2013). Patrocínio na perspectiva do organizador de eventos esportivos. Revista Intercontinental de Gestão Desportiva, 3(2), 106-118.

Close, A. G., \& Lacey, R. (2013). Fit matters? Asymmetrical impact for effectiveness on sponsors and event marketers. Sport Marketing Quarterly, 22(2), 71.

Contursi, E.B. (2003). Patrocínio. Rio de Janeiro: Sprint, 2003.

Crescitelli, E., Mattar, M., \& Silva, A. (2005). O efeito das ações de patrocínio sobre o top of mind: um estudo exploratório do mercado brasileiro. Revista Eletrônica de Gestão de Negócios, 1(2), 1-15.

Rocha, A. L. P., \& Campos, S. F. (2010). Uma avaliação das características mais relevantes em projetos de patrocínio de eventos e sua relação com os objetivos almejados. Revista Eletrônica de Administração, 16(1), 194-223.

Danylchuk, K. E., \& MacIntosh, E. (2009). Food and non-alcoholic beverage sponsorship of sporting events: the link to the obesity issue. Sport Marketing Quarterly, 18(2), 69.

Dees, W., Bennett, G., \& Villegas, J. (2008). Measuring the effectiveness of sponsorship of an elite intercollegiate football program. Sport Marketing Quarterly, 17(2), 79.

Dhurup, M., \& Rabale, E. M. (2012). Spectators' perceptions of official sponsors in the FIFA 2010 World Cup and purchase intentions of sponsors products or brands: FIFA World Cup. African Journal for Physical Health Education, Recreation and Dance, 18(1), 139150 .

Eagleman, A. N., \& Krohn, B. D. (2012). Sponsorship awareness, attitudes, and purchase intentions of road race series participants. Sport Marketing Quarterly, 21(4), 210.

Fernandez, M. (2011) A indústria do esporte no Brasil. In Instituto Illuminatus (Org.). Encontro de gestores de esporte e lazer. São Paulo: Instituto Illuminatus.

Ferrari, T. D., \& Silveira, R. B. (2009). Pra qual time você torce? O Marketing e as Estratégias de Patrocínio Esportivo. Seminários em Administração: Empreendedorismo e inovação, 20.

Grynberg, C. A., \& da Rocha, A. L. P. (2010). Modelos de mensuração do recall de patrocínio esportivo nos consumidores: uma aplicação sobre os patrocinadores da seleção brasileira de futebol. REAd-Revista Eletrônica de Administração, 16(3), 564-588. 
Hawkins, D. I., \& Mothersbaugh, D. L. (2018). Comportamento do consumidor: construindo a estratégia de marketing. Elsevier Brasil.

Keller, K.L.; \& Machado, M. (2006). Gestão estratégica de marcas. SãoCPaulo: Pearson Prentice Hall.

Koo, G. Y., Quarterman, J., \& Flynn, L. (2006). Effect of perceived sport event and sponsor image fit on consumers' cognition, affect, and behavioral intentions. Sport Marketing Quarterly, 15(2).

Kuzma, J. R., Veltri, F. R., Kuzma, A. T., \& Miller, J. J. (2003). Negative corporate sponsor information: The impact on consumer attitudes and purchase intentions. International Sports Journal, 7(2), 140.

Leocádio, A. L., Silvestre, A. C. D., Portela, D. Z., Nakajima, L. Y., \& Silva, T. D. (2007). Marketing esportivo no campeonato brasileiro de futebol: relação entre empresas investidoras e times patrocinados. Seminários em Administração, 10 (3), 32-36.

MacIntosh, E., Nadeau, J., Seguin, B., O'Reilly, N., Bradish, C. L., \& Legg, D. (2012). The role of mega-sports event interest in sponsorship and ambush marketing attitudes. Sport Marketing Quarterly, 21(1), 43.

Mason, K. (2005) How corporate sport sponsorship impacts consumer behavior. The Journal of American Academy of Business, 7 (1), 32-35.

Mattar, M. F. (2007). Tomada de decisão em ações de patrocínio esportivo: análise descritiva do processo decisório e critérios de seleção em empresas patrocinadoras no Brasil (Tese de doutorado, Universidade de São Paulo).

McDaniel, S. R., \& Heald, G. R. (2000). Young consumers' responses to event sponsorship advertisements of unhealthy products: Implications of schema-triggered affect theory. Sport Management Review, 3(2), 163-184.

McKelvey, S., Sandler, D., \& Snyder, K. (2012). Sport participant attitudes toward ambush marketing: An exploratory study of ING New York City Marathon runners. Sport Marketing Quarterly, 21(1), 7.

Melo Neto, F.P. (2003). Marketing de patrocínio. Sprint.

Mielli, J. F., \& Mantovani, D. M. N. (2014). Copa do Mundo 2014 no Brasil: um estudo das atitudes e envolvimento do espectador com o evento. Podium Sport, Leisure and Tourism Review, 3(3), 1-11.

Moraes, I. F. (2017). Marketing esportivo no futebol: Produçao e publicaçao académica brasileira. REVISTA INTERCONTINENTAL DE GESTÃO DESPORTIVA-RIGD, 7(2), 193213. 
Park, M., Yoh, T., Choi, Y. S., \& Olson, M. W. (2011). Consumer attitudes toward the Paralympic Games and purchase intentions toward corporate sponsors of the Paralympic Games: market segmentation strategy. Journal Venue \& Event Management, 3(2), 2-15.

Parolini, P. L., \& Rocco Júnior, J. A. (2016). PATROCÍNIO ESPORTIVO NO BRASIL: UMA ANÁLISE QUALITATIVA DA PRODUÇÃO ACADÊMICA BRASILEIRA SOBRE O TEMA. REVISTA INTERCONTINENTAL DE GESTÃO DESPORTIVARIGD, 6(1), 103-118.

Pimentel, J. G. D. M. (2011). A relevância do profissionalismo no futebol e os impactos da credibilidade dos dirigentes na obtenção de patrocício (Tese de doutorado).

Porto, R. B. (2010). Atitude do Consumidor: Estrutura dos Modelos Teóricos. Revista Brasileira de Marketing, 9(2), 41-58.

Pozzi, L.F. (1998). A grande jogada: teoria e prática do marketing esportivo. São Paulo: Globo.

Reams, L., Eddy, T., \& Cork, B. C. (2015). Points of attachment and sponsorship outcomes in an individual sport. Sport Marketing Quarterly, 24(3), 159.

Rocha, A. L. P., \& Campos, S. F. (2010). Uma avaliação das características mais relevantes em projetos de patrocínio de eventos e sua relação com os objetivos almejados. REAdRevista Eletrônica de Administração, 16(1), 194-223.

Scharf, E. R. (2010). O patrocínio do futebol como ferramenta do marketing esportivo para a construção de brand awareness. Economia \& Gestão, 10(23), 80-99.

SOARES, L. L. S., DELIZA, R., \& GONÇALVES, E. B. (2008). Escalas atitudinais utilizadas em estudos de consumidor: tradução e validação para a língua portuguesa. Alimentos e Nutrição Araraquara, 17(1), 51-64.

Zaharia, N., Biscaia, R., Gray, D., \& Stotlar, D. (2016). No more "good" intentions: Purchase behaviors in sponsorship. Journal of Sport Management, 30(2), 162-175.

Zardini Filho, C. E. (2017). A Influência do Patrocínio Esportivo na Atitude sobre Marcas. REMark: Revista Brasileira de Marketing, 16(2), 194-206.

Zem, C. A., \& Machado, J. R. (2009). O marketing de patrocínio esportivo no contexto das instituições educacionais. Revista de Administração da Unimep-Unimep Business Journal$B 2,2(2)$.

Zunino, R. (2006). Comportamento de Compra de Torcedores de Clubes de Futebol: Um Estudo da Aquisição de Produtos dos Patrocinadores. II EMA-Encontro de Marketing da ANPAD. Rio de Janeiro/RJ, 3. 